\title{
Characterization of materials and structures by the photothermal method
}

C. Fort

CEA-CESTA, Service Physique Expérimentale, BP. 2, 33114 Le Barp, France

\begin{abstract}
An experimental set-up based on the photothermal method is described for the simultaneous measurement of thermal effusivity and thermal diffusivity of materials. It is used to determine the radial thermal diffusivity of anisotropic materials or the thermal diffusivity of thin films, by the converging thermal wave technique.
\end{abstract}

\section{INTRODUCTION}

The technique we discuss here is quite appropriate for thin films, or for anisotropic materials with a thermal radial diffusivity $a_{\mathrm{r}}$ that is very different from the thermal normal diffusivity $\mathrm{a}_{\mathrm{z}}$. For instance, for pyrocarbon we get $a_{\mathrm{r}} / \mathrm{a}_{\mathrm{Z}}>170$. In this paper we present a technique for measuring the radial thermal diffusivity $\mathrm{a}_{\mathrm{r}}=\lambda_{\mathrm{T}} / \rho \mathrm{C}$ where $\rho$ denotes the mass density of the material, $\lambda$ is the thermal conductivity and $C$ is its specific heat. The radial diffusivity $a_{r}$ is measured using a method proposed by Cielo et al. The principle is as follows : the surface of the cylindrical sample is heated in an annular shape, and the temperature rise in the center of the annulus is recorded $(1,2)$. From this experimental thermogram, $a_{r}$ can be evaluated using a method that will be presented later, under the assumptions that : the medium is finite, there are no convection heat losses at the surface of the medium, the energy deposit on the medium is of short duration in comparison with the characteristic radial diffusion time.

\section{EXPERIMENTAL APPARATUS}

The experimental apparatus is shown in Fig. 1. The heating source is a pulsed flash lamp (made with five concentric flash lamps, diameter $230 \mathrm{~mm}$, pulse duration $\tau=0.52 \mathrm{~ms}$, energy $10000 \mathrm{~J} / \mathrm{m}^{2}$ ). The heat pulse is uniform over the entire front surface, for the measurement of the thermal diffusivity and the thermal effusivity. For the measurement of radial thermal diffusivity the heat pulse has an annular shape. The surface temperature is monitored by an infrared $\mathrm{HgCdTe}$ detector $(8-12 \mu \mathrm{m})$ focused with a mirror objective on the center of the non-irradiated disk region on the front side for $\left(a_{r}\right)$ measurement. The back surface temperature is monitored by an other infrared $\mathrm{HgCdTe}$ detector in the same way. For $\left(a_{r}\right)$ measurement, a plane I.R. mirror is placed behind the sample at a short distance from its rear side to set to zero the radiative heat losses of this side. On the front side, an opaque insulation pipe screwed on to the mirror objective, is used in order to simulate an annular heat pulse. The external diameter of the tube $(15 \mathrm{~mm})$ represents the non-irradiated disk region and the hole inside the tube ( $4 \mathrm{~mm}$ ) represents the measurement area. With this system, the measurement area is perfectly concentric with the non-irradiated disk region.

\section{MODELLING AND PRINCIPLE OF THE METHOD FOR $\left(a_{r}\right)$ MEASUREMENT}

The modelling of the experiment is made clear from the sketch in Fig. 2. The cylindrical sample (radius $R_{S}$ ), of finite thickness, (l), is impinged on its front side by an annular heat flux that is assumed uniformly irradiated between $R_{i}$ and $R_{s}$. The different sides are subjected to convective heat losses characterized by the following heat exchange coefficients $\left(h_{0}, h_{1}, h_{R}\right)$. 
The thermograms computed at the center of the sample are shown in Fig. 3. If the "deflectivity D" : $\mathrm{D}=\mathrm{a}_{\mathrm{r}} 1^{2} / \mathrm{a}_{\mathrm{z}} \mathrm{R}_{\mathrm{s}}^{2}$ is less than 0.02 , there is mainly a radial heat flow $\left({ }^{3}\right)$.

Thus, if the surface heat losses are negligible, the thermal radial diffusivity can be calculated by : $\mathrm{a}_{\mathrm{r}}=\mathrm{K} \frac{\mathrm{R}_{\mathrm{i}}^{2}}{\mathrm{t}_{1 / 2}}\left(\mathrm{~m}^{2} / \mathrm{s}\right) \quad$ with $\mathrm{K}=-0.330 \frac{\mathrm{R}_{\mathrm{i}}}{\mathrm{R}_{\mathrm{s}}}+0.419$ and $\mathrm{D} \leq 0.02, \mathrm{R}_{\mathrm{i}} / \mathrm{R}_{\mathrm{s}} \geq 3$ where $R_{i}$ is the radius of the non irradiated disk region on the front side and $t_{1 / 2}$ is the time it takes the sample to heat to half of the maximum temperature at the center on the front surface. The variation of $K$ is shown in Fig. 4.

\section{MEASUREMENT OF RADIAL DIFFUSIVITY}

We compare the results obtained by the standard flash method. In such a technique, a sample of known thickness is irradiated on one side by a short heat pulse, and the thermal history on the front side and on the opposite side are simultaneously recorded with infrared detectors (Fig. 1.) $\left({ }^{4}\right)$. With the thermogram of the irradiated surface we can identify thermal effusivity $(5)$ :

$\mathrm{b}=\sqrt{\lambda \rho \mathrm{C}}, \quad \mathrm{b}=\frac{\mathrm{Q}}{\Delta \mathrm{T}(\mathrm{t}) \sqrt{\pi \mathrm{t}}} \quad \mathrm{Q}:$ is the pulse energy of density (Dirac heat flux)

and thermal diffusivity :

$\mathrm{a}_{\mathrm{z}}=\frac{\lambda}{\rho \mathrm{C}}, \quad \mathrm{a}_{\mathrm{z}}=\frac{1}{\pi} \frac{1^{2}}{\mathrm{t}_{\mathrm{rp}}} \quad \mathrm{t}_{\mathrm{rp}}:$ transition time between the semi infinite and the finite media

With the thermogram of the non-irradiated surface we can identify thermal diffusivity $(6)$ :

$\mathrm{a}_{\mathrm{z}}=0.139 \frac{\mathrm{1}^{2}}{\mathrm{t}_{1 / 2}}$

We have used a titanium sample (isotropic material). The results witch have been obtained are presented below. The experimental and the calculated curves are shown in Fig. 5.

\begin{tabular}{|c|c|c|}
\hline $\mathrm{b} \quad\left(\mathrm{J} / \mathrm{m}^{2} \mathrm{~K} \mathrm{~s} \mathrm{~s}^{1 / 2}\right)$ & 6670 & \\
\hline$a_{z}=\frac{1}{\pi} \frac{1^{2}}{t_{r p}}\left(m^{2} / s\right)$ & $7.910^{-6}$ & \\
\hline$a_{z}=0.139 \frac{1^{2}}{t_{1 / 2}}\left(\mathrm{~m}^{2} / \mathrm{s}\right)$ & $8.010^{-6}$ & \\
\hline$a_{r}=K \frac{R_{i}^{2}}{t_{1 / 2}}\left(m^{2} / s\right)$ & $7.710^{-6}$ & $\mathrm{D}=1.610^{-3}$ \\
\hline
\end{tabular}

For anisotropic materials, the temperature excursion at the center of the non-irradiated disk region on the front side (Fig. 6) is very different. There are simultaneously two heat flows, a radial one and an axial one. The experimental thermogram obtained with a pyrocarbon sample is shown in Fig. 6 $\left(\mathrm{a}_{\mathbf{r}} / \mathrm{a}_{\mathbf{z}}>170\right)$.

$$
a_{z}=1.510^{-6} \mathrm{~m}^{2} / \mathrm{s}, \quad a_{r}=2.610^{-4} \mathrm{~m}^{2} / \mathrm{s} \quad \text { and } \quad \mathrm{D}=2.5
$$

\section{CONCLUSIONS}

In summary, the converging thermal wave technique has been used to measure the radial diffusivity on thin films or anisotropic materials. Typical accuracies in the measurement of thermal diffusivity are in the range of $\pm 2 \%$ for $\mathrm{D} \leq 0.02$ and for small heat losses.

\section{REFERENCES}

[1] Cielo P., Utracki L. A., Lamontagne M., Can. J. Phys. Vol.64 (1986) 1172-1177

[2] Enguehard F., Boscher D., Deom A., Balageas D.L., Mater. Sci. Eng. B5 (1990) 127-134.

[3] Guyonvarch G., DEA ISITEM Nantes (1991).

[4] Fort C., Roux J.M., Guidon M., Soci. Fran. Therm. (S.F.T.) Congrès annuel (1988)

[5] Balageas D. L., Krapez J.C., Cielo P., J. Appl. Phys. Vol.59 (1986).

[6] Parker W. J., Jenkins R. J., Butler C. P., Abbott G. L., J. Appl. Phys. Vol.32 (1961). 


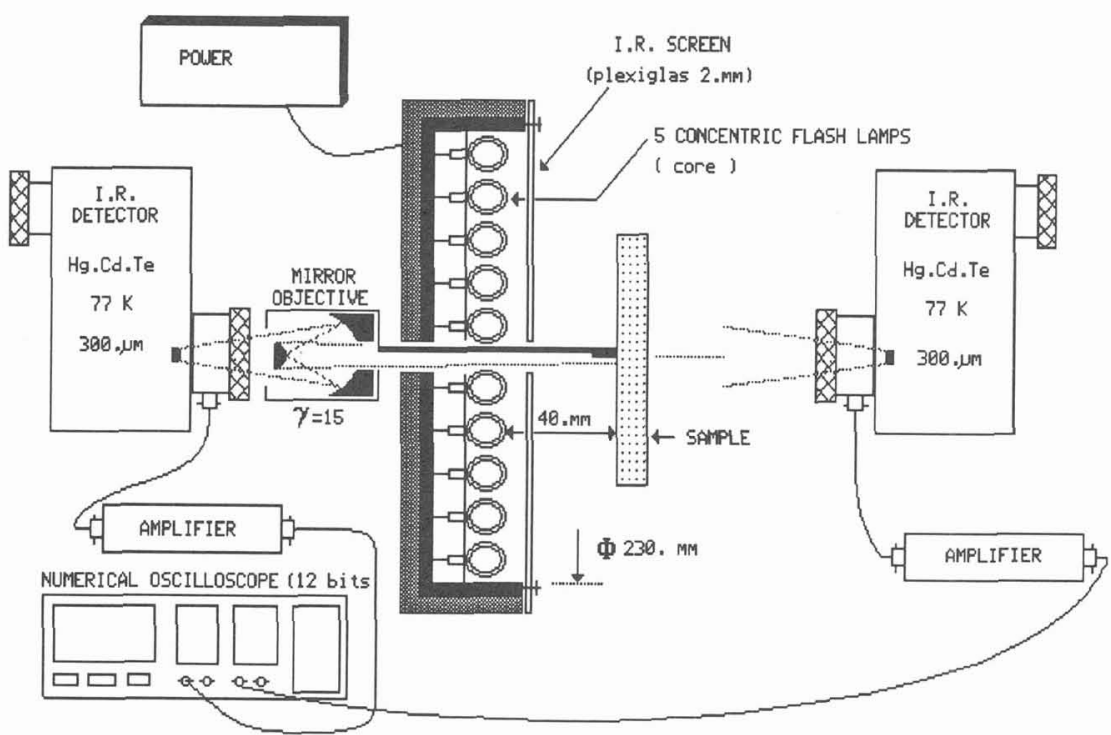

Fig. 1 : Experimental set-up.

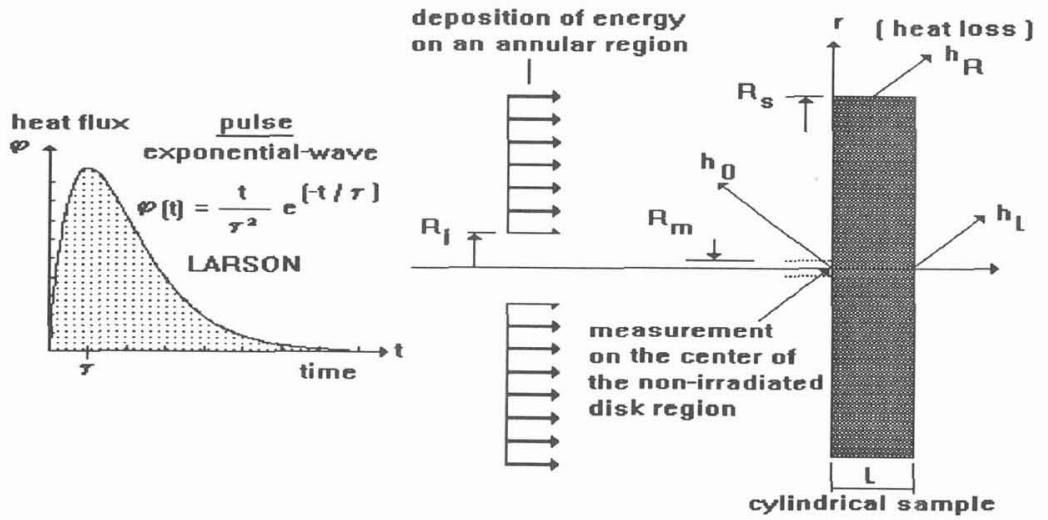

Fig. 2 : Schematic diagram of the experimental set-up for the measurement of radial diffusivity. $\mathbf{R}_{\mathrm{S}}$ : sample radius, $\mathbf{R}_{\mathbf{i}}$ : irradiated radius, $\mathbf{R}_{\mathrm{m}}$ : measurement radius.

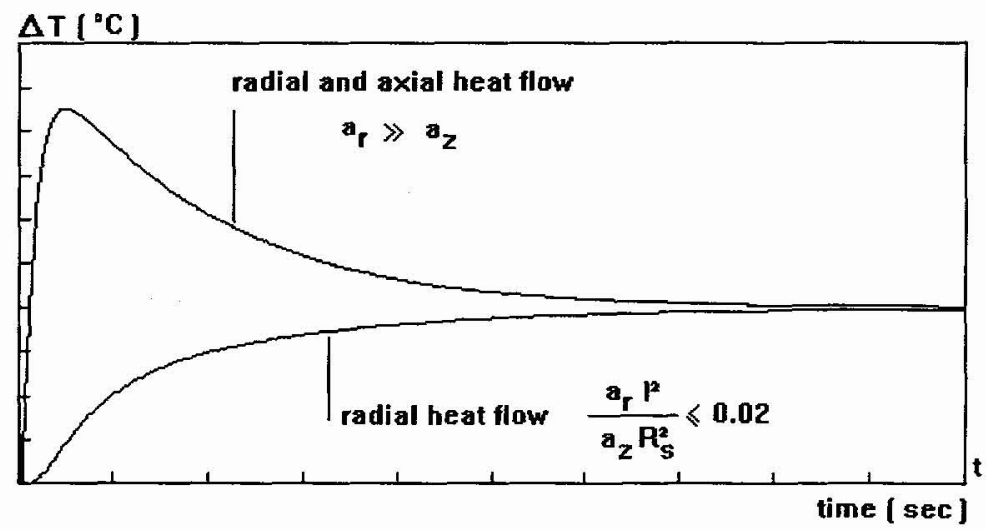

Fig. 3 : Temperature excursion at the center of the non-irradiated disk region on the front side. 


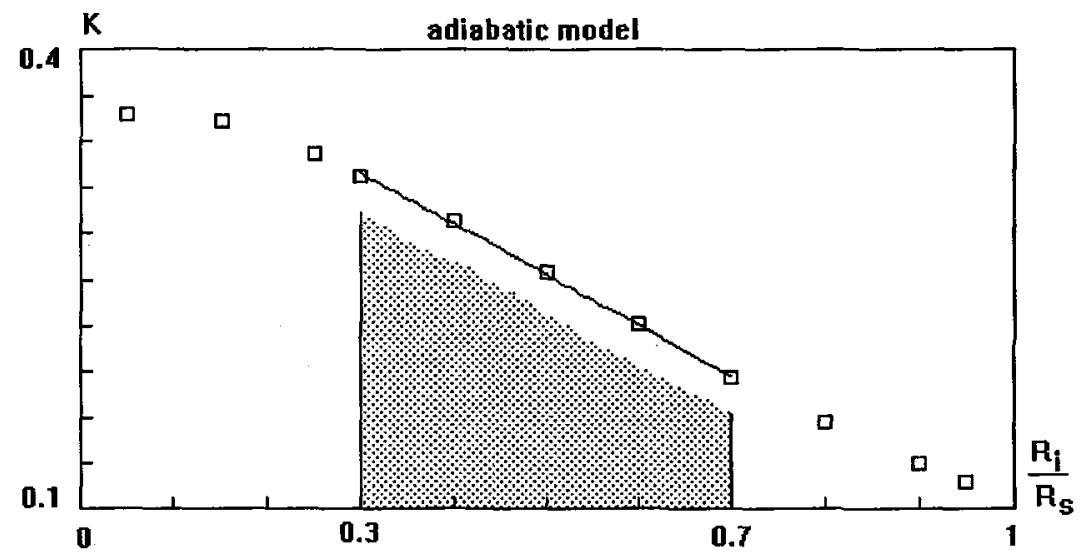

Fig. 4 : Determining the radial diffusivity $\left(a_{r}\right)$.

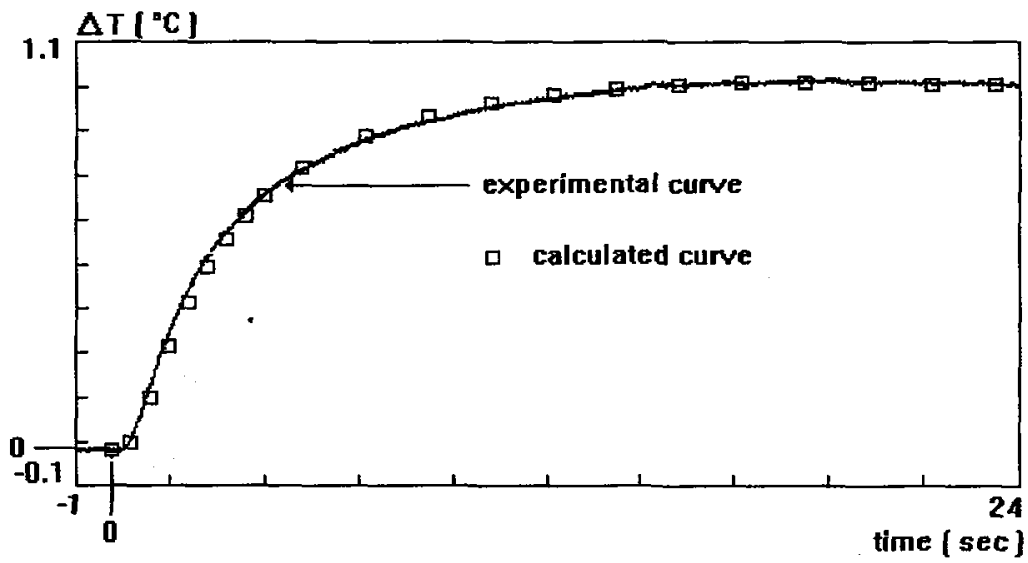

Fig. 5 : Thermogram of the non-irradiated region on the front side. Titanium sample ( $1=1 \mathrm{~mm})$. Isotropic material. $R_{\mathrm{s}}=25 \mathrm{~mm}, \mathrm{R}_{\mathrm{i}}=7.5 \mathrm{~mm}, \mathrm{R}_{\mathrm{m}}=2 \mathrm{~mm}$.

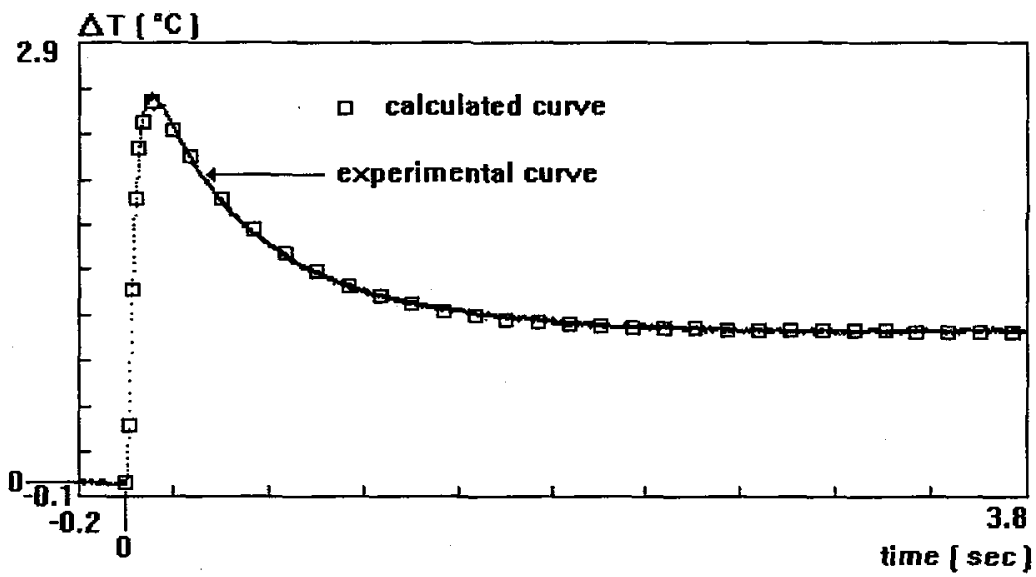

Fig. 6 : Thermogram of the non-irradiated region on the front side. Pyrocarbon sample $(1=3 \mathrm{~mm})$. Anisotropic material. $R_{\mathrm{s}}=25 \mathrm{~mm}, \mathrm{R}_{\mathrm{i}}=7.5 \mathrm{~mm}, \mathrm{R}_{\mathrm{m}}=2 \mathrm{~mm}$. 